\title{
Tuberculosis primaria de la glándula suprarrenal: comunicación de dos casos
}

Adrenal gland primary tuberculosis: report of two cases

\author{
Natalia Coras ${ }^{1, a}$, José Somocurcio ${ }^{1, b}$, Cristian Aguilar ${ }^{1,0}$ \\ Servicio de Anatomía Patológica, Hospital Nacional Edgardo Rebagliati Martins, EsSalud, Lima, Perú. \\ ${ }^{a}$ Médico Asistente; ${ }^{b}$ Médico Jefe; ${ }^{c}$ Médico Residente.
}

\begin{abstract}
Resumen
En muchas áreas del mundo, la tuberculosis tiene gran prevalencia y sigue siendo la principal causa de insuficiencia suprarrenal. Clínicamente, esta enfermedad permanece sin sintomatología durante sus primeros estadios, debido a que la pérdida de la función adrenal suele ser progresiva; los síntomas tienen aparición gradual e insidiosa, en su mayoría inespecíficos. Presentamos el caso de dos mujeres con cuadro clínico de insuficiencia adrenal primaria crónica, con afección bilateral de ambas glándulas suprarrenales y resultado histopatológico de infección activa por tuberculosis.
\end{abstract}

Palabras clave: Glándula suprarrenal, tuberculosis suprarrenal, insuficiencia suprarrenal.

\section{Abstract}

Tuberculosis is highly prevalent and is still currently the leading cause of adrenal insufficiency in many areas of the world. Clinically this disease remains asymptomatic during early stages, but due to adrenal function loss symptoms become insidious and gradual, mostly unspecific. We report two cases of female patients with clinical symptoms of chronic bilateral primary adrenal insufficiency with active tuberculosis infection by histopathology.

Keywords: Adrenal glands, adrenal tuberculosis, adrenal insufficiency.

\section{An Fac med. 2013;74(3):221-6}

\section{INTRODUCCIÓN}

Durante muchos años la tuberculosis (TBC) ha sido considerada como la principal causa de insuficiencia suprarrenal de tipo infecciosa. En la actualidad, la causa más frecuente de insuficiencia adrenal a nivel mundial es la adrenalitis autoinmunitaria (1). La adrenalitis de tipo tuberculosa es la causa más frecuente en países en vías de desarrollo, en 5 a $6 \%$ de casos de tuberculosis activa las glándulas están afectadas ${ }^{(2)}$.

El compromiso de la glándula adrenal suele permanecer clínicamente latente durante décadas y en el momento del diagnóstico, cuando aparecen sus manifestaciones clínicas, la destruc- ción de las mismas puede llegar hasta 90\%. En cuanto a los síntomas de la insuficiencia adrenal crónica son poco específicos y no se les distingue de otras causas; dependen del grado de severidad de la deficiencia de las hormonas producidas en estas glándulas (cortisol, aldosterona y andrógenos).

El diagnóstico se confirma con la determinación hormonal en sangre, que muestra concentraciones matutinas bajas de cortisol sérico, asociadas a concentraciones elevadas de ACTH; también pueden estar disminuidas las concentraciones de aldosterona y de sulfato de dehidroepiandrosterona (DHEA-S). Cuando se presentan dudas, se hace uso de algunas pruebas dinámicas, como la medición seriada de cortisol sérico durante la prueba de hipoglicemia insulínica o la prueba de estimulación endovenosa con ACTH, las que demuestran la falta de elevación de los niveles de cortisolemia. Se confirma la causa tuberculosa mediante demostración del bacilo, a través de biopsia, baciloscopia, cultivo y reacción en cadena de la polimerasa (PCR) ${ }^{(3,4)}$. Algunos autores han descrito características radiológicas tomográficas compatibles con AdTBC (Criterios de Nerup, 1974) ${ }^{(5)}$.

Se presenta dos casos de adrenalitis de tipo tuberculoso estudiados y diagnosticados en el Departamento de Anatomía Patológica y el servicio de 
Endocrinología de nuestro hospital. El objetivo de nuestro estudio fue evaluar las características clínicas, imagenológicas e histopatológicas de la insuficiencia adrenal de tipo tuberculoso.

\section{CASO 1}

Paciente mujer de 47 años con antecedente de exposición a TBC hacía 10 años (hermana con tuberculosis pleural), sin otros antecedentes patológicos y quirúrgicos de importancia. Un año antes de su ingreso, presentó pigmentación de piel y mucosas asociada a dolor abdominal tipo cólico a predominio de flanco izquierdo, con náuseas, vómitos y deposiciones liquidas esporádicas. Una semana antes del ingreso tuvo debilidad generalizada e hiporexia, por lo cual acudió al servicio de urgencias por la intensidad de síntomas. Al examen físico presentaba hipotensión arterial, hiperpigmentación de piel en las regiones periocular, labial, malar, palmas de manos y plantas de pies, así como en nudillos de dedos y mucosa oral; el abdomen era blando, depresible, algo doloroso, sin tumoraciones palpables; el resto del examen no fue contributorio.

Tuvo biometría hemática normal, glucosa $84 \mathrm{mg} \%$, urea y creatinina normales, sodio $135 \mathrm{mEq} / \mathrm{L}$, potasio 5,6 $\mathrm{mEq} / \mathrm{L}$, VSG $55 \mathrm{~mm} / \mathrm{h}$, cortisol (am) $<1,00 \mathrm{ug} / \mathrm{dL}$, aldosterona $<28 \mathrm{pg} / \mathrm{mL}$, DHEAS <15,0 ug/dL, ACTH (am): $180 \mathrm{pg} / \mathrm{mL}$ (VN: 0-45), PRL 0,71 ng/ $\mathrm{mL}$, IGF-1 182,7 ng/mL, TSH 0,37 uUI/ mL, T4L 1,03 ng/dL, AbTPO-AbTG negativos, FSH 1,75 mUI/mL, LH 2,50 $\mathrm{mUI} / \mathrm{mL}$, E2 $84 \mathrm{pg} / \mathrm{mL}, \mathrm{BK}$ en esputo y lavado gástrico negativos, anticuerpos anti-adrenales negativos (IFI), ELISAHIV 1 y 2 negativo, CEA 0,9 ng/mL, Ca125 24,9 U/mL, Ca19-9 9,3 U/mL, Ca15-3 7,2 U/mL. No se realizó PPD.

La ultrasonografía abdominal reveló glándula suprarrenal derecha de $22 \mathrm{x}$ $18 \mathrm{~mm}$ y la izquierda de $30 \times 26 \mathrm{~mm}$, con formaciones nodulares, hipoecoicas, de características heterogéneas. La radiografía de tórax no mostró hallazgos patológicos. La tomografía tóraco-
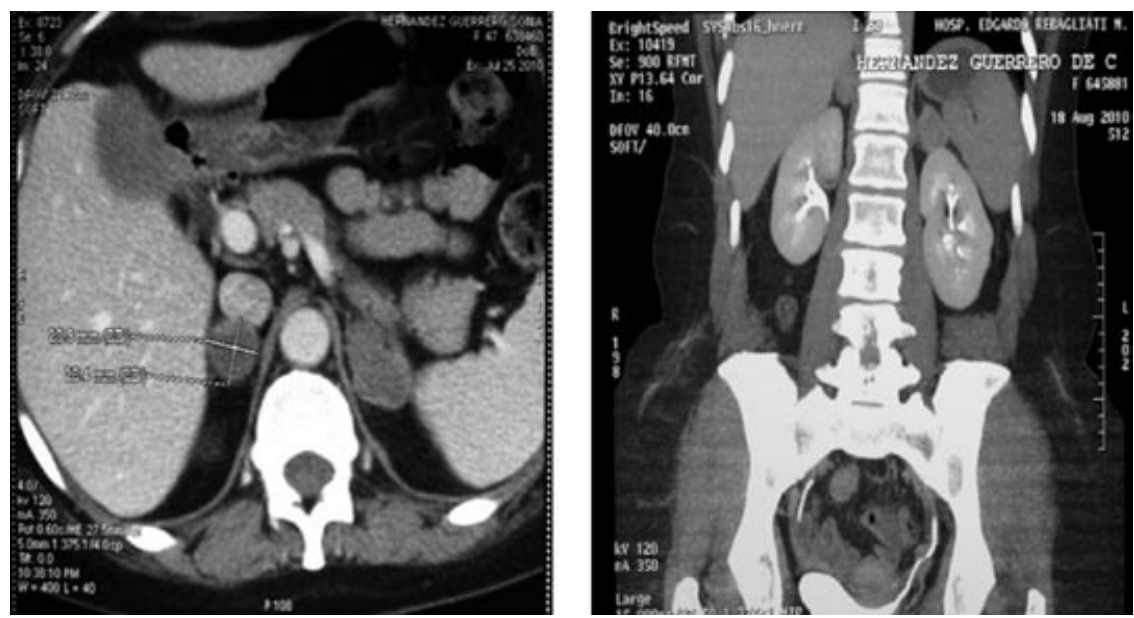

Figura 1. En la tomografía axial computarizada, ambas glándulas adrenales con lesión focal ocupativa, nodular, mixta, con componente quístico.

abdominal (figura 1) confirmó la presencia de lesiones nodulares en ambas glándulas suprarrenales, con escasa vascularización en la fase contrastada, sin evidencia de adenopatías retroperitoneales. No había presencia de lesiones a nivel pulmonar.

Ante el cuadro de insuficiencia adrenal y la presencia de lesiones tumorales en ambas glándulas adrenales, se decidió realizar la resección laparoscópica de la glándula derecha, resección que terminó siendo bilateral por decisión del cirujano urólogo.

En la macroscopia, la glándula suprarrenal derecha medía $5,5 \times 3 \times 2 \mathrm{~cm}$, la izquierda $6 \times 4,8 \times 2,8 \mathrm{~cm}$, parduscas, cubiertas por tejido adiposo. A los cor- tes, había lesión tumoral en la derecha de $1,3 \times 1,2 \mathrm{~cm}$, en la izquierda de $1,8 \mathrm{x}$ $1,4 \mathrm{~cm}$, con necrosis central, color pardo amarillento, heterogénea, que comprometía casi toda la glándula (figura 2). A la microscopia se observó proceso inflamatorio crónico, granulomatoso, con extensas aéreas de necrosis caseosa, en relación a tuberculosis adrenal bilateral (figura 3). La tinción histoquímica con técnica de Zielh Neelsen para bacilos ácido alcohol resistentes fue positiva $(+/+++)$ (figura 4$)$. No se realizó cultivo ni PCR.

La paciente recibió tratamiento combinado para tuberculosis, con el primer esquema, según las normas nacionales. Posteriormente, presen-
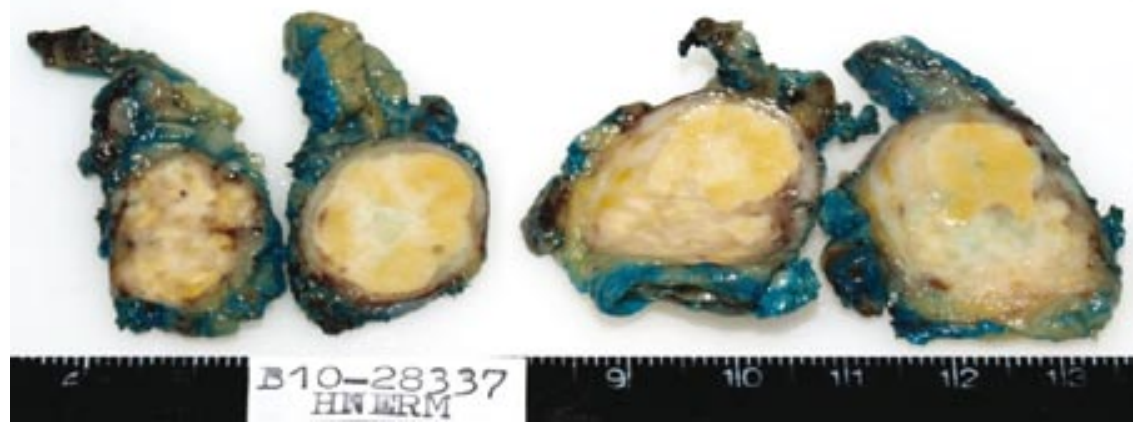

Figura 2. En la macrofotografía, ambas glándulas suprarrenales con lesiones nodulares, con necrosis central, heterogéneas, que comprometían casi toda la glándula. 


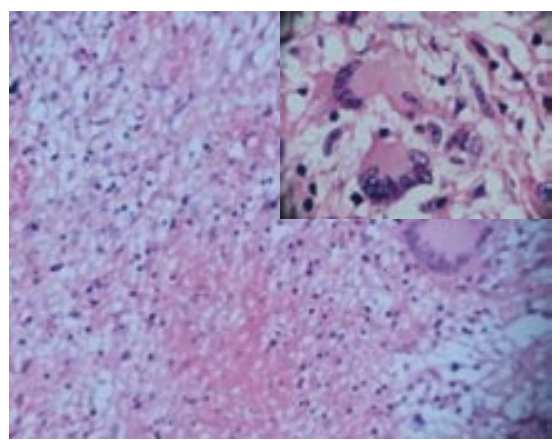

Figura 3. En la microfotografía, se observa proceso inflamatorio crónico granulomatoso, con extensas aéreas de necrosis, con células gigantes multinucleadas (HE- 40X)

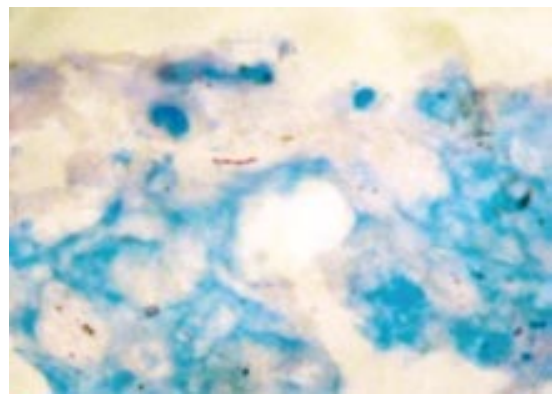

Figura 4. La tinción histoquímica con técnica de Ziehl Neelsen para bacilos ácido alcohol resistentes fue positiva $(+/+++)$ (Bk-100x).

to reacción adversa a rifampicina, por lo cual se cambió a ciprofloxacino. El tratamiento para insuficiencia adrenal fue inicialmente parenteral, con hidrocortisona $150 \mathrm{mg}$ a $300 \mathrm{mg}$ diarios, y posteriormente y hasta el momento de esta comunicación con prednisona $(5$ a $15 \mathrm{mg} /$ día $)$ y fludrocortisona $(0,05 \mathrm{a}$ $0,1 \mathrm{mg} /$ día). La paciente presentó evolución clínica favorable.

\section{CASO 2}

Paciente mujer de 75 años procedente de Iquitos, con antecedente de hepatitis viral B e histerectomía por mioma uterino hacía 20 años. Sin exposición conocida a TBC, desde hacía 6 meses presentaba hiperpigmentación de piel y mucosas, asociada a dolor abdominal intermitente, hiporexia, debilidad generalizada y disminución de peso de $8 \mathrm{~kg}$. Fue transferida al servicio de urgencia de nuestro hospital, con cuadro caracterizado por náuseas, vómitos de contenido alimentario cada tres días, dolor abdominal tipo cólico en epigastrio, intermitente y sensación de debilidad generalizada. Al examen físico mostraba presión arterial normal, ortostatismo, hiperpigmentación a nivel de mucosa oral, lengua y piel, a predominio de palmas, rostro y zonas de flexión; resto sin alteraciones.

La biometría hemática fue normal, glucosa $117 \mathrm{mg} / \mathrm{dL}$, urea y creatinina normales, sodio $126 \mathrm{mmol} / \mathrm{L}$, potasio $5,8 \mathrm{mmol} / \mathrm{L}$, PCR $1,4 \mathrm{mg} / \mathrm{dL}$, VSG 76 $\mathrm{mm} / \mathrm{h}$, DHL $1751 \mathrm{U} / \mathrm{L}, \mathrm{Ca} 125$ 35,1 U/ $\mathrm{mL}, \mathrm{Ca} 19-9<2 \mathrm{U} / \mathrm{mL}$, Ca15-3 7,9 U/ $\mathrm{mL}$, AFP 2,5 ng/mL, CEA 2,5 ng/mL, ANA negativo (IFI), TSH 3,27 uUI/ $\mathrm{mL}$, T4L 0,92 ng/dL, AbTPO-AbTG negativos, DHEAS $<15 \mathrm{ug} / \mathrm{dL}$, androstenediona $0,58 \mathrm{ng} / \mathrm{mL}$, cortisol 1,06 ug/ dL, ACTH 211,8 pg/mL, aldosterona $<28 \mathrm{pg} / \mathrm{mL}$, PRL $6,41 \mathrm{ng} / \mathrm{mL}$, ELISAHIV 1 y 2 negativos, BK en esputo, orina y jugo gástrico negativos, cultivo de BK en esputo negativo. No se realizó PPD y la radiografía de tórax no mostró hallazgos patológicos.

La ultrasonografía abdominal reveló una glándula suprarrenal derecha de $27 \times 13 \mathrm{~mm}$ y la izquierda de $34 \mathrm{x}$ $30 \mathrm{~mm}$, con formaciones nodulares,

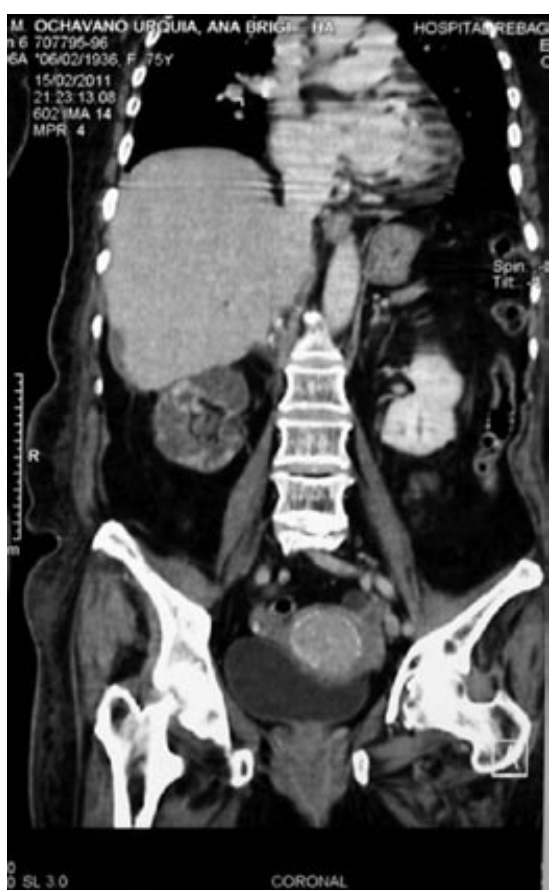

Figura 5. Tomografía axial computarizada. Se observa aumento del tamaño de ambas glándulas adrenales.

hipoecoicas, con bordes regulares. La tomografía tóraco-abdominal mostró en pulmones pequeñas densidades de aspecto cicatricial, algunas bronquiectasias, mediastino sin tumoraciones ni adenopatías. Incremento de las dimensiones de ambas adrenales con imagen

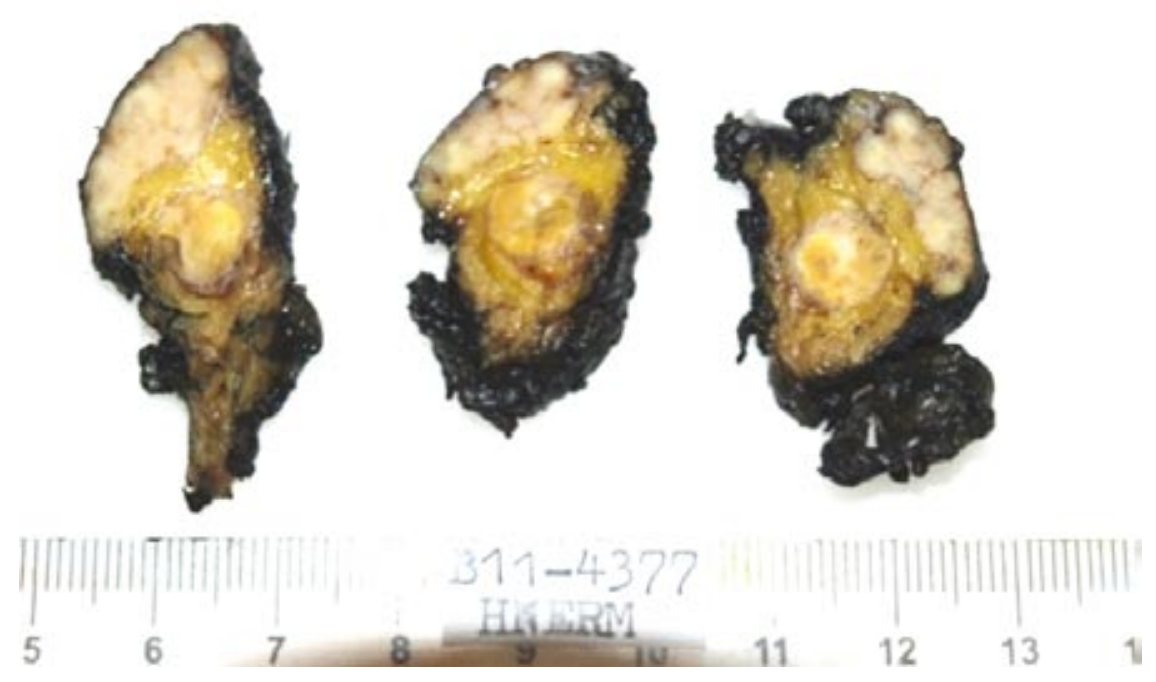

Figura 6. Macrofotografía con lesión nodular pardo amarillento en glándula adrenal derecha. 


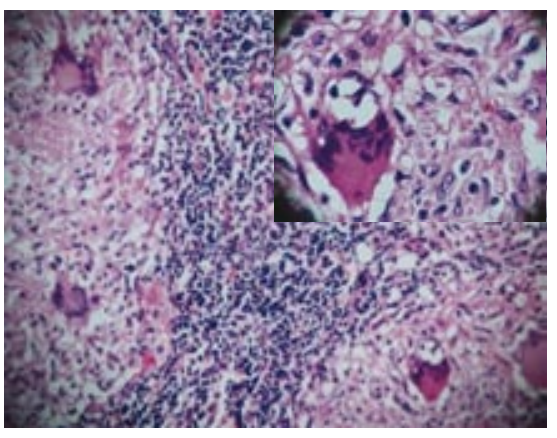

Figura 7. En la microfotografía, se observa proceso inflamatorio crónico granulomatoso con compromiso de la estructura adrenal, con extensas aéreas de necrosis caseosa (HE-40X).

nodular en el lado izquierdo, de 19,8 x $14,6 \mathrm{~mm}$, y en el lado derecho imagen ovoidea bilobulada que medía $36,4 \mathrm{x}$ 29,6 mm; no se evidenció calcificaciones ni adenopatías retroperitoneales; resto del abdomen sin alteraciones significativas (figura 5).

Ante la sospecha de probable origen neoplásico, se decidió realizar la resección de la glándula derecha por vía laparoscópica.

En la macroscopia, la glándula suprarrenal derecha midió 5 × 4,8 x 2,8 $\mathrm{cm}$, pardo amarillenta, cubierta parcialmente por tejido adiposo. A los cortes seriados se apreció lesión nodular pardusca con punteado amarillento circunscrito (figura 6). A la microscopia se observó proceso inflamatorio crónico granulomatoso de origen tuberculoso,

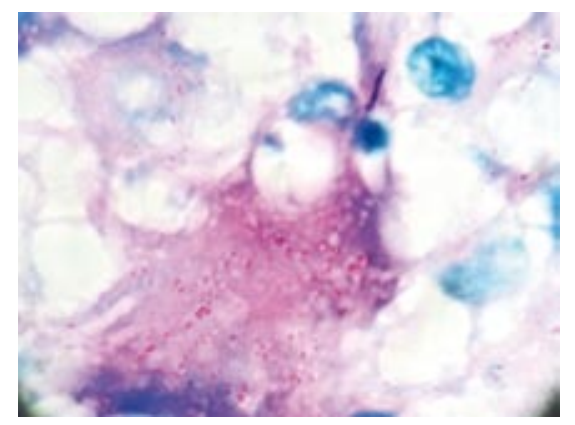

Figura 8. La tinción histoquímica con técnica de Ziehl Neelsen resultó positiva (+/+++) para bacilos ácido alcohol resistentes (Bk-100x). que comprometía casi toda la estructura de la glándula adrenal, con extensas aéreas de necrosis caseosa (figura 7). La tinción histoquímica con la técnica de Zielh Neelsen resultó positiva $(+/+++)$ para bacilos ácido alcohol resistentes (figura 8).

El caso fue catalogado como tuberculosis adrenal primaria, BK positivo. La paciente recibió tratamiento con el primer esquema antituberculoso, según norma nacional, con evolución clínica favorable. Al momento de la presente comunicación recibe reemplazo glucocorticoide y mineralocorticoide (prednisona 5 a $7,5 \mathrm{mg} /$ día y fludrocortisona 0,05 a $0,1 \mathrm{mg} / \mathrm{día}$, por vía oral) y parenteral con dosis mínimas de andrógenos IM.

\section{DISCUSIÓN}

La incidencia de la tuberculosis extrapulmonar, como sitio primario de enfermedad, es variable según el lugar donde se publique. Varía en los últimos años de 10 a 26\%, siendo más frecuente en los ganglios linfáticos $(10,4 \%)$, pleural $(4,7 \%)$, osteoarticular, entre otros ${ }^{(6)}$.

La adrenalitis tuberculosa es de rara presentación. Ocurre solo en $6 \%$ de los pacientes con tuberculosis activa. A pesar de la reducción de este porcentaje, el compromiso adrenal sigue siendo de difícil diagnóstico y se sabe que muchos casos no son notificados y solo son hallados en estudios posmórtem. La OMS predice un incremento continuo de la TBC y se estima que entre 19 y $43 \%$ de la población mundial está infectada por la micobacteria tuberculosis, la mayoría en forma de infección latente ${ }^{(7,8)}$. Esto supone un enorme reservorio de individuos, entre los que son esperables nuevos casos. No se conoce claramente el tropismo del bacilo por las glándulas suprarrenales. Suele permanecer clínicamente latente durante décadas y, en el momento del diagnóstico, cuando aparecen sus manifestaciones clínicas, la destrucción de las mismas puede llegar hasta $90 \%$ y en la mayoría de los casos es bilateral, pero asimétrica ${ }^{(9)}$.
En los países en vías de desarrollo, la causa principal continúa siendo la infecciosa, específicamente por $\mathrm{My}$ cobacterium tuberculosis, así como se informa una prevalencia de $47 \%$ en la India y $34 \%$ en Sudáfrica ${ }^{(1,10)}$. En Perú, carecemos de cifras epidemiológicas, por las escasas series no publicadas de insuficiencia adrenal de tipo tuberculoso como sitio primario. Otras causas menos frecuentes son las metástasis suprarrenales, fármacos, infecciones, enfermedades infiltrativas y procesos vasculares ${ }^{(11)}$.

Durante la fase aguda de la infección de la glándula suprarrenal, esta sufre un aumento de tamaño, que puede o no dar el cuadro de la insuficiencia suprarrenal. Posteriormente, a lo largo de los años, la glándula sufrirá un proceso de atrofia y calcificación, con pérdida progresiva de la reserva hormonal ${ }^{(12)}$, como se observó en los pacientes estudiados.

Los síntomas de insuficiencia suprarrenal crónica son poco específicos. Consisten en astenia, anorexia y pérdida de peso. Es importante pensar en esta enfermedad ante un paciente con un síndrome general. La hipotensión se da en $88 \%$ de los pacientes y va acompañada de síntomas de ortostatismo. Otros síntomas menos frecuentes son náuseas, vómitos, dolor abdominal, diarrea o estreñimiento, apetencia por la sal, apatía, depresión, mialgias, entre otros ${ }^{(13,14)}$. La hiperpigmentación cutánea ocurre en 86 a $94 \%{ }^{(15)}$, como signo relativamente más específico; en nuestros pacientes se presentó como compromiso cutáneo y mucoso. La molécula de ACTH en las células del lóbulo intermedio de la hipófisis es precursora de la hormona estimulante de los melanocitos (alfa- MSH), que promueve la pigmentación de la piel; y dado que la ACTH comparte con alfa-MSH el grupo de aminoácidos con esta capacidad estimulatoria, genera hiperpigmentación de piel y mucosas\%. ${ }^{(16)}$

El diagnóstico es confirmado por concentraciones muy bajas de cortisol sérico $(<3 \mathrm{ug} / \mathrm{dL})$ y demás hormonas 
corticales, la elevación de ACTH; y cuando existen dudas, la falta de elevación de la cortisolemia ( $>20 \mathrm{ug} / \mathrm{dL}$ ) durante la prueba de hipoglicemia insulínica o la administración endovenosa de ACTH \% ${ }^{(4)}$; nuestros pacientes presentaron niveles de cortisol muy disminuidos.

La sospecha de tuberculosis puede darse por la realización de la prueba dérmica, como el PPD (no disponible en nuestro centro), u otros exámenes no disponibles aún en nuestro medio, como son las pruebas basadas en la producción de gamma interferón por los linfocitos $\mathrm{T}$ en respuesta a antígenos específicos, los cuales están presentes en todas las cepas patógenas de micobacteria tuberculosis y micobacteria bovis ${ }^{(15)}$. También puede considerarse la negatividad de los anticuerpos antiadrenales (realizado solo en una paciente a pesar de su sensibilidad baja) y se confirma mediante la demostración del bacilo, con biopsia, baciloscopia, cultivo y PCR ${ }^{(3,4)}$. En nuestros casos, presentaron positividad para el bacilo en tejido directo.

La tomografía computarizada (TC) es una técnica diagnóstica muy importante para el estudio de la patología suprarrenal, por su alta resolución. Existen diversos estudios que han tratado de establecer una serie de criterios de imagen específicos que permitan distinguir entre los tumores primarios y la afección tuberculosa de las glándulas suprarrenales ${ }^{(17,18)}$.

Aunque debe tenerse en cuenta que los hallazgos pueden variar dependiendo del estadio evolutivo de la enfermedad. Así, en fases agudas del fallo por tuberculosis u otros procesos infecciosos, se produce un aumento del tamaño de las suprarrenales, observables con técnicas de imagen debido a la infiltración de células inflamatorias con formación de granulomas. En formas agudas-subagudas, se ha descrito la observación en la TC de hipertrofia glandular, típicamente bilateral, generalmente con zonas necróticas atenuadas y realce periférico. Posteriormente, el remplazo progresivo por fibrosis y nódulos caseosos conduce en aproximadamente dos años a la disminución del tamaño de las adrenales, mientras que en las fases muy evolucionadas las glándulas pueden estar atróficas y presentar calcificaciones, lo cual sugiere afectación tuberculosa ${ }^{(19,20)}$. Nuestros casos presentaron atrofia bilateral de ambas adrenales, por lo que podrían corresponder a una fase evolucionada de la enfermedad.

La combinación de bilateralidad y de captación central baja con realce periférico proporciona una sensibilidad y una especificidad elevadas para el diagnóstico de tuberculosis adrenal. Las calcificaciones también orientan a una etiología tuberculosa, aunque solo $15 \%$ de los tumores primarios puede presentarlas ${ }^{(19)}$. Por el contrario, las tumoraciones unilaterales de aspecto sólido suelen ser otros tumores ${ }^{(19,21)}$

En el compromiso autoinmune, suelen mostrar un tamaño disminuido, y en los casos de metástasis o infiltraciones muestran un aumento de tamaño, con configuración alterada y borramiento de los límites con las estructuras vecinas. La biopsia percutánea o la resección estarían indicadas ante aumentos de tamaño de la glándula, con o sin calcificaciones, a menos de que existan datos fehacientes de tuberculosis activa en otro órgano o de neoplasia conocida o de metástasis detectadas en otros sitios ${ }^{(17,21)}$. Ante la sospecha de neoplasia maligna de las glándulas adrenales, se decidió realizar la resección quirúrgica de las mismas. Por otra parte, los estudios analíticos en nuestros casos descartaron hipersecreción hormonal, y revelaron una insuficiencia suprarrenal primaria, que con frecuencia acompaña a la tuberculosis adrenal, principalmente cuando se ha producido ya una destrucción del 90\% del tejido cortical. En los dos casos estudiados, en el estudio histopatológico de las glándulas adrenales se observó la pérdida casi total de la estructura glandular.

En conclusión, la insuficiencia suprarrenal primaria debe sospecharse ante un paciente con síntomas inespecíficos, hiperpigmentación mucocutánea difusa progresiva, hallazgo de cortisolemia muy disminuida o la falta de elevación de las pruebas dinámicas descritas, niveles bajos de otras hormonas corticoadrenales; mientras que la sospecha de la etiología dependerá de las características de cada paciente, hallazgos inmunológicos y en las imágenes -como la tomografía abdominal- compromiso de ambas las glándulas suprarrenales, imágenes con compromiso bilateral, sin afectación en otros órganos. En países como el nuestro, debemos considerar estudiar las causas infecciosas, específicamente tuberculosis, cuando se encuentren las características epidemiológicas y los hallazgos en las imágenes con contraste, aunque en ocasiones solo el estudio histológico resultara definitivo. Si bien es cierto que la adrenalitis tuberculosa es una causa potencialmente tratable, la existencia confirmada o sospechada de TBC activa requiere, además, un tratamiento precoz con tuberculostáticos, para la recuperación parcial o total de la función adrenal. Esto último no es posible en la mayoría de casos ${ }^{(22)}$, como sucedió en nuestras pacientes, quienes reciben terapia de reemplazo hasta el momento de esta comunicación.

\section{REFERENCIAS BIBLIOGRÁFICAS}

1. Ten S, New M, Maclaren N. Clinical review 130 : Addison's disease 2001- J Clin Endocrinol Metabol. 2001;86:2909-22.

2. Agarwal G, Bhatia E, Pandey R, Jain SK. Clinical profile and prognosis of Addison's disease in India. Natl Med J India. 2001;14:23-5.

3. Lanzas-Prieto JM, Guate-Ortiz JL, GonzálezTuero J, et al. Adrenal tuberculosis Diagnosis using polymerase chain reaction. Arch Esp Urol. 1997;50(7):802-4.

4. Paz-Ibarra JL. Manual de Pruebas Dinámicas en Endocrinología. Sociedad Peruana de Endocrinología. 2009.

5. Montoya Álvarez T, Pavón de Paz I, Olivar J, Peñalver D, Iglesias P. Dificultades diagnósticas en un caso de enfermedad de Addison de origen tuberculoso. Endocrinol Nutr. 2007;54(5):279-82.

6. Tuberculosis in New York City, 1999: Information summary. New York City Department of Health. 2000.

7. Corbett EL, Watt CJ, Walker N. The growing burden of tuberculosis: global trends and interactions with the HIV epidemic. Arch Intern Med. 2003;163:100921. 
8. Kim JH, Langston AA, Galis HA. Miliary tuberculosis: epidemiology, clinical manifestations, diagnosis, and outcome. Rev Infect Dis. 1990;12:583-90

9. Lam KY, Lo CY. A critical examination of adrenal tuberculosis and a 28-year autopsy experience of active tuberculosis Clin Endocrinol (Oxf). 2001;54(5):633-9.

10. Soule S. Addison's disease in Africa: a teaching hospital experience- ClinEndocrinol (Oxf). 1999;50(1):115-20.

11. Caron P, Chabannier MH, Cambus JP, Fortenford $F$, Otal $P$, Suc JM. Definitive adrenal insufficiency due to bilateral adrenal hemorrhage and primary antiphospholipid syndrome. J Clin Endocrinol Metab. 1998;83(5):1437-9.

12. Sanford J, Favour C. The interrelationships between Addison's disease and active tuberculosis: a review of 125 cases of Addison's disease. Ann Intern Med. 1976;65:56-62.

13. Barnard C, Kanani R, Friedman JN. Her tongue tipped us off CMAJ. 2004;171(5):451.

14. Vilardell-Latorre E. Enfermedades de las glándulas suprarrenales: Farreras-Rozman, editor. Medicina Interna. 13a. ed. Barcelona: Mosby Doyma libros, 1995;2116-43.
15. Garcia Pascual L, Simó Canonge R, Mesa Manteca J, Obiols Alfonso G, Segura García A, González Atienza J. Enfermedad de Addison: un estudio clínico-epidemiológico de 45 casos. Rev Clin Esp. 1990;187(2):49-52.

16. Thomas B, Fitzpatrick. Dermatologia en Medicina General, $7^{\text {a }}$ edición. Editorial Medica Panamericana. 2008.

17. Yang ZG, Guo YK, Li Y, Min PQ, Yu JQ, et al. Differentiation between tuberculosis and primary tumors in the adrenal gland: evaluation with contrastenhanced CT. EurRadiol. 2006;16:2031-6.

18. Guo YK, Yang ZG, Li Y, Deng YP, Ma ES, Min PQ, et al. Uncommon adrenal masses: CT and MRI features with histopathologic correlation. Eur J Radiol. 2007;62:359-70.

19. Bacarizo $P$, Checa MR, Suárez Llanos $P$, Hernández $L$, Jara Albarrán A. Evolución radiológica en el seguimiento de la adrenalitis tuberculosa. Endocrinol Nutr. 2006;53:510-4.

20. Buxi TB, Vohra RB, Sujatha, Byotra SP, Mukherji S, Daniel M. CT in adrenal enlargement due to tuberculosis: a review of literature with five new cases. Clin Imaging. 2002;26:102-5.
21. Halperin Rabinovich I. Diagnóstico de la enfermedad de Addison. Clínica, radiologia, análisis y biopsia ¿Todos para uno o uno para todos? Med Clin (Barc). 1993;101:132-5.

22. Baruah Manash. Sub-clinical addison's disease. Indian J Endocrinol Metab. 2012;16(Suppl 2):S176S177.

Artículo recibido el 18 de diciembre de 2012 y aceptado para publicación el 1 de abril de 2013.

Los autores declaran que no existen conflictos de intereses.

\section{Correspondencia:}

Dra. Natalia Coras Álvarez.

Servicio de Anatomía Patológica- Hospital Nacional Edgardo Rebagliati Martins

Av. Velasco Alvarado 123. V.E.S, Lima-Perú.

Teléfono: (01)493-2036

Correo electrónico: nataliacoras@gmail.com. 